Research Article

\title{
Day-to-Day Dynamic Multivehicle Assignment: Deterministic Process Models
}

\author{
Giulio E. Cantarella and Chiara Fiori iD \\ Department of Civil Engineering, University of Salerno, Fisciano 84084, Italy \\ Correspondence should be addressed to Chiara Fiori; cfiori@unisa.it
}

Received 3 December 2020; Revised 5 February 2021; Accepted 4 March 2021; Published 26 March 2021

Academic Editor: Manuel De la Sen

Copyright (c) 2021 Giulio E. Cantarella and Chiara Fiori. This is an open access article distributed under the Creative Commons Attribution License, which permits unrestricted use, distribution, and reproduction in any medium, provided the original work is properly cited.

\begin{abstract}
In the near future, transportation systems modelers and planners will likely be challenged by more complex scenarios. This is due to the different types of vehicles that include different (i) powertrains (conventional, hybrid, electric, etc.), (ii) ownerships (privately-owned vs. shared vehicles), and (iii) levels of automation (from human-driven to fully autonomous). All these different vehicle types compete for the same arcs and jointly participate to congestion. Thus, existing methods for travel demand assignment to a transportation network, the main tools for transportation systems analysis to support transportation project assessment and evaluation, need to be extended to cope with mixed traffic. In this paper, deterministic process models for day-today dynamic multivehicle assignment are presented, including fixed-point models for equilibrium assignment as a special case. Vehicle types may be distinguished with respect to several parameters, such as flow equivalence coefficient, occupancy factor, cost equivalence coefficient, and behavioral parameters. Results of an application to a toy network are also discussed showing that advanced vehicles (AVs) may or may not have a positive effect of equilibrium stability.
\end{abstract}

\section{Introduction}

Technologies and prototypes for advanced vehicles (AVs), such as connected, automated, autonomous vehicles, or electric and hybrid ones, both for transportation of people or goods are fast developing. Still, the time needed to turn the existing stock of (mostly privately-owned) traditional vehicles (TVs) into (possibly shared) advanced vehicles will last several years during which mixed traffic is expected. Indeed, according to current estimates, transition to widespread fully autonomous and electric cars will require at least 20 years. Meanwhile, enhancements of existing tools of Traffic and Transportation Theory are required by transportation systems modelers and planners. Thus, methods for travel demand assignment to a transportation network, the main tools for transportation systems analysis to support transportation project assessment and evaluation, need to be extended to cope with mixed traffic.

Equilibrium assignment was introduced by Wardrop (1952) [1]; afterwards, Stochastic User Equilibrium based on random utility theory (RUT) was introduced by Daganzo and Sheffi (1977) [2]. Equilibrium assignment may effectively be formulated through fixed-point (FP) models (introduced by Daganzo, 1983 [3], through the use of inverse cost function) as shown by the general framework in Cantarella (1997) [4]. This modeling approach implemented in several commercial software packages is the most used in practical applications.

Methods for day-to-day (or interperiodic) dynamic assignment (introduced for transportation systems analysis by Horowitz, 1984 [5]) play a central role in advanced transportation system analysis, since they allow analysing and forecast equilibrium stability and fluctuations around it, as a result of past events. Indeed, even though exactly one equilibrium flow and cost patterns exist, the system state, flow and cost patterns, may evolve towards the equilibrium or towards another kind of attractor.

The stability analysis can be addressed through deterministic process (DP) models, derived from discrete-time nonlinear dynamic systems theory [6,7]. Moreover, the equilibrium analysis does not allow analysing transients after demand and/or supply changes, or obtaining a statistical 
description of the system state evolution over time, i.e., means, modes, moments, and, more generally, frequency distributions, this kind of analysis requiring stochastic process (SP) models (introduced for transportation systems analysis by Cascetta, 1987 [8], 1989 [9]). A first unifying framework was proposed by Cantarella and Cascetta (1995) [10]. Additional details on SP are available (e.g., [11-13]).

A general theory of travel demand assignment including static and dynamic modeling approaches has recently been proposed in the book by Cantarella et al. (2019) [14], which is a comprehensive reference for mathematical details as well as references.

In a recent paper, Cantarella and Di Febbraro (2017) [15] showed how existing fixed-point (FP) models for equilibrium assignment can be extended to transportation networks where several types of vehicles, including, for instance, AVs and TVs, compete for the same arcs and jointly participate to congestion. Cantarella et al. [16, 17] provided sufficient conditions for fixed-point existence uniqueness, specified algorithms for large-scale applications, analysed conditions for their convergence, and discussed some large-scale examples.

This paper proposes DP models for day-to-day dynamic multivehicle assignment. Different vehicle types may be given by the following:

Fossil fuelled vs. electrical energy

Ownership, say privately owned vs. (operator-owned) shared vehicles

Level of automation, from human-driven to fully autonomous

Others

According to the proposed modeling approach, if the route choice behavior is described by applying the Random Utility Theory, vehicle type may be distinguished through different parameters regarding dispersion of perceived utility (in the choice function), weights of attributes (in the utility function), etc. Vehicle types may also be distinguished with respect to the occupancy factor, that is, the average number of users on-board, the flow equivalent factor, that is, the relative effect on capacity, and the cost equivalent factor.

In Section 2, the adopted multivehicle modeling approach is described, briefly reviewing the relevant background. Then, in Section 3, some simple examples considering two types of vehicles, TVs and AVs moving on a toy network, are discussed showing the effecting of increasing the AVs proportion. Major findings and research perspectives are discussed in Section 4.

The main original contributions of this paper are as follows:

Deterministic process models for day-to-day dynamic multivehicle assignment, including fixed-point models for equilibrium assignment as a special case

Vehicle types may be distinguished with respect to several parameters as shown below

Comparison between moving average and exponential smoothing filters for modeling user memory and learning
An application to a toy network showing that, even in very simple networks, AVs may or may not have a positive effect of equilibrium stability

\section{Multivehicle Assignment}

In this section, the adopted modeling approach is described, and; it is consistent with the Six Equation Assignment Modeling (SEAM) and the Two Equation Assignment Modeling approaches proposed in Cantarella et al. (2019) [14], where mathematical details are discussed. User category is not explicitly introduced in the following to simplify notations.

2.1. Assignment to Uncongested Networks. Connections are described by an oriented graph. Parking facility can easily be considered. Each origin and each destination are modeled through a further node connected to the main network through connecting arcs. Users are distinguished with respect to o-d pair $i$ they are travelling from/to and type of used vehicle $m$. The reference vehicle type (RVT) has index $m=1$; usually, privately owned human-driven fossil fuelled passenger cars are used as RVT.

2.1.1. Basic Definitions and Equations. The cost per each vehicle type $m$ is given by an affine transformation of the RVT cost:

$$
\mathbf{c}_{m}=\chi_{m} * \mathbf{c}+\mathbf{c}_{\mathrm{Z} m} \forall m
$$

where, with reference to vehicle type $m, \mathbf{c}_{\mathbf{Z} m} \geq \mathbf{0}$ is the arc specific cost vector per vehicle type; $\mathbf{c} \geq \mathbf{0}$ is the arc cost vector, common to all vehicle types; $\chi_{m}>0$ is the cost equivalence coefficient, modeling, for example, different on-board comfort, speeds, etc. with $\chi_{1}=1$ (for further details, see Section $2.4)$; and $\mathbf{c}_{m} \geq \mathbf{0}$ is the arc cost vector per vehicle type.

The route costs can be obtained from the corresponding arc costs through an affine transformation from the arc space to the route space defined by the transpose of arc-route incidence matrix:

$$
\mathbf{w}_{i m}=\mathbf{B}_{i m}{ }^{\mathrm{T}} \cdot \mathbf{c}_{m}+\mathbf{w}_{Z i m} \forall i m,
$$

where, with reference to o-d pair $i$ and vehicle type $m$, $\mathbf{w}_{\text {Zim }} \geq \mathbf{0}$ is the route specific or nonadditive cost vector, such as not arc-based tolls or fares; $\mathbf{B}_{i m}$ is the arc-route incidence matrix; and $\mathbf{w}_{i m} \geq \mathbf{0}$ is the route cost vector; arc and route (generalized transportation) costs are assumed measured by a common unit, usually travel time or money, through duly homogenization of different attributes, such as the value of time, not explicitly introduced to simplify notations.

The (systematic) utility function for o-d pair $i$ and vehicle type $m$ is specified through a linear transformation (almost always in research analysis as well as in practical applications):

$$
\mathbf{v}_{i m}=-\psi_{m} \mathbf{w}_{i m} \forall i m,
$$

where, with reference to o-d pair $i$ and vehicle type $m, \psi_{m}>0$ is the utility scale parameter, such that the term $\psi_{i} \mathbf{w}_{i}$ is dimensionless, and $\mathbf{v}_{\text {im }}$ is the route systematic utility vector. 
Route choice behavior for o-d pair $i$ and vehicle type $m$ can be modeled by applying any discrete choice modeling theory so that route choice proportions depend on route utility:

$$
\mathbf{p}_{i m}=\mathbf{p}_{i m}\left(\mathbf{v}_{i m} ; \boldsymbol{\theta}_{m}\right) \forall i m,
$$

where, with reference to o-d pair $i$ and vehicle type $m, \mathbf{p}_{\text {im }}>0$ is the route choice proportion vector and $\boldsymbol{\theta}_{m}>0$ is the route choice function parameter vector, with $\theta_{m 1}$ being the scale factor (if present), as in a Logit choice model.

Most often, the choice function $\mathbf{p}_{\text {im }}(\bullet)$ in (4) is derived from Random Utility Theory (RUT). Examples are the wellknown models belonging to Logit family or Probit and the more recent Gammit and Weibit. If the parameters of the perceived utility pdf do not depend on systematic utility values, the resulting choice function, called invariant, is monotone increasing with symmetric semidefinite positive Jacobian.

Flow conservation ensures that flows of all routes connecting the o-d pair $i$ using vehicle type $m$ sum up to demand flow:

$$
\mathbf{h}_{i m}=d_{i} q_{i m} \mathbf{p}_{i m} \forall i m,
$$

where, with reference to o-d pair $i$ and vehicle type $m, q_{i m} \geq 0$ is the proportion of the total demand flow per vehicle type and o-d pair; $d_{i m} \geq 0$ is the demand flow; and $\mathbf{h}_{i m} \geq 0$ is the route flow vector.

The average number of users on board, possibly different per vehicle type, is introduced to convert demand and route flows measured in users per unit of time into arc flows measured in vehicles per unit of time. Furthermore, a flow equivalence coefficient for each vehicle type needs to be introduced to measure arc flows in RVTs per time unit (and to distinguish the effect on congestion, see Section 2.2.1).

The arc total flows are given by the sum of arc flows plus the arc base flows due to each combination of o-d pair $i$ and vehicle type $m$ over all o-d pairs and vehicle types:

$$
\mathbf{f}=\Sigma_{i} \Sigma_{m} \eta_{i m}\left(\frac{1}{\varphi_{m}}\right) \mathbf{B}_{i m} \mathbf{h}_{i m}+\mathbf{f}_{z},
$$

where, with reference to vehicle type $m, \mathbf{f}_{z} \geq 0$ is the arc base flow vector; $\varphi_{m}>1$ is the occupancy factor, that is, the average number of users on board; $\eta_{m}>0$ is the flow equivalence coefficient, with $\eta_{1}=1$; and $\mathbf{f} \geq 0$ is the arc (total) flow vector.

2.1.2. Arc Flow Function. If the vehicle type demand flows are given. the arc flow function between arc flows and (common) arc costs can be obtained by combining together equations (1)-(6):

$$
\mathbf{f}=\mathbf{f}\left(\mathbf{c} ;\left(\frac{\eta_{m}}{\varphi_{m}}\right), \chi_{m}, \psi_{m}, \boldsymbol{\theta}_{m} \forall m\right)
$$

The arc function (7) models the effects of the route choice behavior at a macroscopic scale. It has values in the arc feasibility set $S_{f}$, which is nonempty (if the network is connected), compact, and convex. The arc flow function (7) differs from the arc flow function with one vehicle type, since several parameters have been introduced to distinguish the vehicle types; see Section 2.4 for further discussion.

The arc function (7) is a linear combination of route choice functions; thus, it is continuous and $c$. differentiable if all the route choice functions are continuous and $c$. differentiable; moreover, it is monotone nondecreasing with symmetric semi-definite negative Jacobian if all the route choice functions are invariant.

The arc flow function (7) can be computed with or without explicit route enumeration even for very large-scale applications through algorithms derived from graph theory.

2.2. Equilibrium Assignment to Congested Networks. In congested networks, arc costs are assumed depending on arc flows due to congestion. Equilibrium assignment models search for mutually consistent arc flows and costs.

2.2.1. Arc Cost Function. The arc cost function models the driving behavior at a macroscopic scale:

$$
\mathbf{c}=\mathbf{c}(\mathbf{f} ; \boldsymbol{\kappa}) \geq 0, \quad \forall \mathbf{f} \in S_{f},
$$

$\boldsymbol{\kappa}>\mathbf{0}$ is the vector of the arc capacities, with entries $\kappa_{a}$, say the maximum flow that may traverse arc $a$, measured consistently with arc flows in RVTs per time unit; in most functions, the arc cost actually depends on the ratio between the arc flow and the capacity, $f_{a} / \kappa_{a}$; in this case, the capacity plays the role of arc flow scale factor.

Other parameters of the arc cost function are not explicitly introduced.

The cost function is called separable; the cost of each arc $a, c_{a}$, depends on the corresponding flow, $f_{a}$ only, nonseparable, otherwise. All usually adopted cost functions are continuous and $c$. differentiable; in most cases, they are $s$. increasing monotone too; often, but not always, they have symmetric Jacobian, as it occurs for separable cost functions with diagonal Jacobian.

2.2.2. Fixed-Point Models. The above-described arc flow functions (7) can be combined with the arc cost function (8) to specify fixed-point (FP) models for equilibrium assignment:

$$
\begin{aligned}
& \mathbf{c}^{*}=\mathbf{c}\left(\mathbf{f}^{*}\right) \in \mathbf{c}\left(S_{\mathbf{f}}\right), \\
& \mathbf{f}^{*}=\mathbf{f}\left(\mathbf{c}^{*} ;\left(\frac{\eta_{m}}{\varphi_{m}}\right), \chi_{m}, \psi_{m}, \boldsymbol{\theta}_{m} \forall m\right) \in S_{f} .
\end{aligned}
$$

Equivalent formulations with respect to flows

$$
\mathbf{f}^{*}=\mathbf{f}\left(\mathbf{c}\left(\mathbf{f}^{*}\right) ;\left(\frac{\eta_{m}}{\varphi_{m}}\right), \chi_{m}, \psi_{m}, \boldsymbol{\theta}_{m} \forall m\right) \in S_{f},
$$

or costs only

$$
\mathbf{c}^{*}=\mathbf{c}\left(\mathbf{f}\left(\mathbf{c}^{*} ;\left(\frac{\eta_{m}}{\varphi_{m}}\right), \chi_{m}, \psi_{m}, \boldsymbol{\theta}_{m} \forall m\right)\right) \in \mathbf{c}\left(S_{f}\right),
$$

are often used in the literature. 
The above FP models are consistent with those discussed by Cantarella and Di Febbraro (2017) [15] and Cantarella et al. $[16,17]$. Existence of equilibrium flows or costs is guaranteed if both the arc cost function and the arc flow function are continuous (if the network is connected). For monotone decreasing arc flow function (7), if the arc cost function (8) is monotone $s$. increasing, uniqueness is guaranteed. The above fixed-point models can be solved for very large-scale applications through algorithms based on the Method of Successive Averages.

2.3. Day-to-Day Dynamic Assignment to Congested Networks. Day-to-day dynamic assignment models try to describe the evolution over time of arc flows and costs.

2.3.1. Further Definitions and Equations. The specification of a model for day-to-day dynamic assignment requires an extension of models for the equilibrium assignment by including submodels of the following:

(i) User memory and learning: how users forecast the level of service that they will experience today, from experience and other sources of information, such as informative systems, about previous days.

(ii) User habit and inertia to change: how users make a choice today, possibly repeating yesterday choice to avoid the effort needed to take a decision, or reconsidering it according to the forecasted level of service.

The arc cost updating relation, modeling user memory and learning, gives the today forecasted route costs with respect to previous day costs. It extends equation (9).

This relation can be specified by an exponential smoothing (ES) filter, say a convex combination of yesterday route forecasted costs and yesterday actual route costs, given by an affine transformation of the yesterday arc costs:

$$
\mathbf{x}^{k}=\beta \mathbf{c}\left(\mathbf{f}^{k-1}\right)+(1-\beta) \mathbf{x}^{k-1}
$$

for given $\mathbf{f}^{0}$, and $\mathbf{x}^{0}=\mathbf{c}\left(\mathbf{f}^{0}\right)$, where $\mathbf{x}^{k-1} \geq 0$ is the forecasted arc cost vector, common to all vehicle types, for day $k-1$; $\beta \in$ ] 0,1 [ is the cost updating parameter, that is, the weight given to yesterday actual costs; in the following, the cost updating parameter $\beta$ is assumed time invariant; it can be different per vehicle type; $\mathbf{f}^{k-1} \geq 0$ is the arc flow vector for day $k-1 ; \mathbf{c}\left(\mathbf{f}^{k-1} ; \boldsymbol{\kappa}\right)$ is the actual arc cost vector, common to all vehicle types, for day $k-1$; and $\mathbf{x}^{k} \geq 0$ is the forecasted arc cost vector, common to all vehicle types, for day $k$.

A model of user memory and learning process with explicitly finite memory depth $\mu$ can be specified through a moving average, MA $(\beta, \mu)$, filter with one parameter $\beta$. At this aim, first the weights of the ES filter are only applied to $\mu$ previous days; thus at day $t$, the summation starts at day $t-\mu$ +1 ; then a scaling factor ensuring that weights $\zeta_{k}$ sum to 1 is applied; the resulting decreasing weights $\zeta_{j}$ for last $\mu$ days are given by

$$
\zeta_{j}=\left(\frac{\beta(1-\beta)^{j-1}}{\left(1-(1-\beta)^{\mu}\right)}\right) \geq 0, \quad \forall j=1,2, \ldots \mu,
$$

where $\mu>1, \mu$ integer, is the memory depth and $\zeta_{j}$ is the weight given to the actual cost occurring in any of the $\mu$ previous days, $\sum_{j} \zeta_{j}=1$.
The resulting (strict) convex moving average $\operatorname{MA}(\beta, \mu)$ filter with one parameter is given by

$$
\mathbf{x}^{k}=\sum_{j=1, \ldots, \mu} \zeta_{j} \mathbf{c}\left(\mathbf{f}^{k-j}\right), \quad \forall k>\mu
$$

with suitable initialization.

The arc flow updating relation, modeling user habit and inertia to change, gives the today arc flow with respect to forecasted costs and previous day flows. It extends equation (10).

This relation can be specified by an exponential smoothing (ES) filter, say a convex combination of yesterday arc flows due to users who do not reconsider their yesterday choice and today arc flows due to users who reconsider their yesterday choice:

$$
\mathbf{f}^{k}=\alpha \mathbf{f}\left(\mathbf{x}^{k-1} ;\left(\frac{\eta_{m}}{\varphi_{m}}\right), \chi_{m}, \psi_{m}, \theta_{m} \forall m\right)+(1-\alpha) \mathbf{f}^{k-1} \forall k,
$$

where $\alpha \in] 0,1$ [ is the choice updating parameter, that is, the proportion of users reconsidering yesterday choice; in the following, the choice updating parameter $\alpha$ is assumed dayinvariant; it can be different per vehicle type.

2.3.2. Deterministic Process Models. The above-described arc flow updating relation (16) can be combined with the arc cost updating relation (13) or (15) to specify deterministic process (DP) models for day-to-day dynamic assignment.

ES/ES DP models are given by the following equations:

$$
\begin{aligned}
\mathbf{x}^{k} & =\beta c\left(\mathbf{f}^{k-1}\right)+(1-\beta) \mathbf{x}^{k-1}, \quad \forall k, \\
\mathbf{f}^{k} & =\alpha \mathbf{f}\left(\mathbf{x}^{k-1} ;\left(\frac{\eta_{m}}{\varphi_{m}}\right), \chi_{m}, \psi_{m}, \boldsymbol{\theta}_{m} \forall m\right)+(1-\alpha) \mathbf{f}^{k-1} \forall k,
\end{aligned}
$$

for given $\mathbf{f}^{0}$, and $\mathbf{x}^{0}=\mathbf{c}\left(\mathbf{f}^{0}\right)$.

The DP model (11-14) can easily be rewritten as a proper Markovian DP by putting equation (13) into (15), but still keeping equation (13). The state variables of DP model (11, 14) are $\left(\mathbf{x}^{k}, \mathbf{f}^{k}\right)$; the updating parameters are $\alpha$ and $\beta$; other parameters are demand flow, $\mathrm{d}$, and any other parameter in the arc flow function and in the arc cost function.

MA/ES DP models are given by the following equations:

$$
\begin{aligned}
& \mathbf{x}^{k}=\sum_{j=1, \ldots, \mu}, \zeta_{j} \mathbf{c}\left(\mathbf{f}^{k-j}\right), \quad \forall k>\mu, \\
& \mathbf{f}^{k}=\alpha \mathbf{f}\left(\mathbf{x}^{k-1} ;\left(\frac{\eta_{m}}{\varphi_{m}}\right), \chi_{m}, \psi_{m}, \boldsymbol{\theta}_{m} \forall m\right)+(1-\alpha) \mathbf{f}^{k-1} \forall k,
\end{aligned}
$$

where

$$
\zeta_{j}=\frac{\beta(1-\beta)^{j-1}}{\left(1-(1-\beta)^{\mu}\right)} \geq 0, \quad \forall j=1,2, \ldots, \mu,
$$

with suitable initialization. 


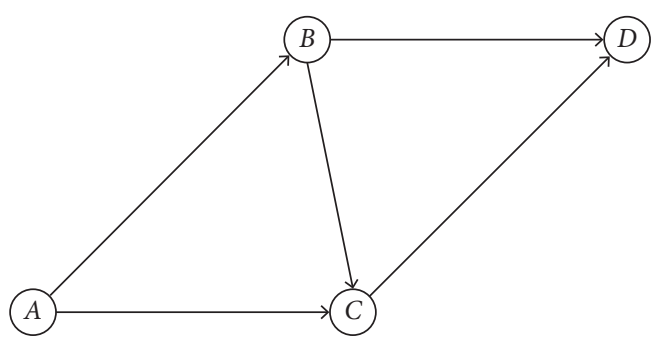

Figure 1: The toy network.

TABLE 1: Parameters of arc cost functions.

\begin{tabular}{lcccc}
\hline Arc & $c_{0 \mathrm{a}}$ & $\kappa_{a}$ & $\nu_{1}$ & \\
\hline 1 & 15 & 2400 & 2.5 & \\
2 & 8 & 3600 & 2.0 & \\
3 & 12 & 2400 & 1.5 & 4 \\
4 & 24 & 3600 & 2.0 & 4 \\
5 & 15 & 3600 & 1.5 & 4 \\
\hline
\end{tabular}

Table 2: Parameters of vehicle types.

\begin{tabular}{lcc}
\hline Type & $\left(\eta_{m} / \mu_{m}\right)$ & $\chi_{m}$ \\
\hline 1 (TVs) & 1.0 & 1.0 \\
2 (AVs) & 0.8 & 0.9 \\
\hline
\end{tabular}

TABLE 3: Bifurcation demand flow, reference scenario $\theta_{\mathrm{TV}}=7.0$.

\begin{tabular}{|c|c|c|c|c|c|c|c|c|}
\hline \multirow[b]{2}{*}{$\begin{array}{l}\text { Scenario } \\
\theta_{\mathrm{TV}}=7.0\end{array}$} & \multicolumn{2}{|c|}{$\mathrm{q}_{m}$} & \multirow{2}{*}{$\begin{array}{c}\text { DP-ES/ } \\
\text { ES } \\
\text { dBif }\end{array}$} & \multirow[b]{2}{*}{$\begin{array}{l}\mu=2 \\
\text { dBif }\end{array}$} & \multicolumn{3}{|c|}{ DP-MA/ES } & \multirow[b]{2}{*}{$\begin{array}{l}\mu=6 \\
\text { dBif }\end{array}$} \\
\hline & TVs & AVs & & & $\begin{array}{l}\mu=3 \\
\text { dBif }\end{array}$ & $\begin{array}{l}\mu=4 \\
\text { dBif }\end{array}$ & $\begin{array}{l}\mu=5 \\
\text { dBif }\end{array}$ & \\
\hline 0 & 1 & 0 & 3917 & 3875 & 3771 & 3917 & 3896 & 3917 \\
\hline
\end{tabular}
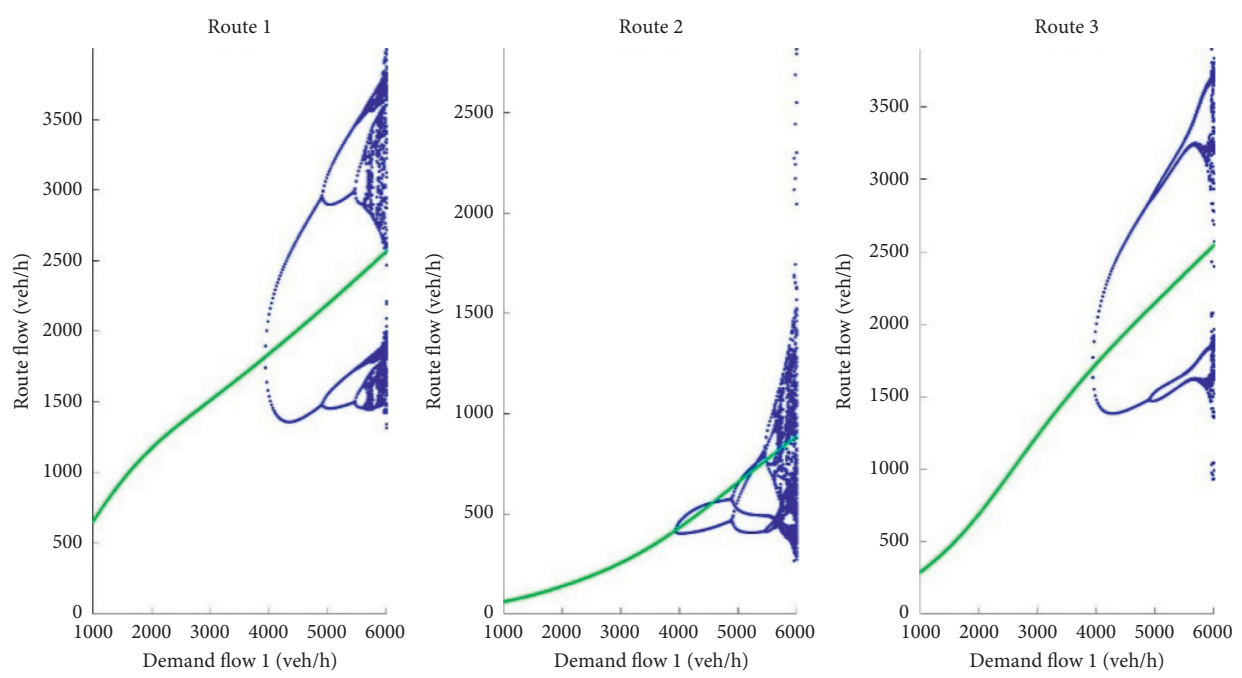

(a)

Figure 2: Continued. 

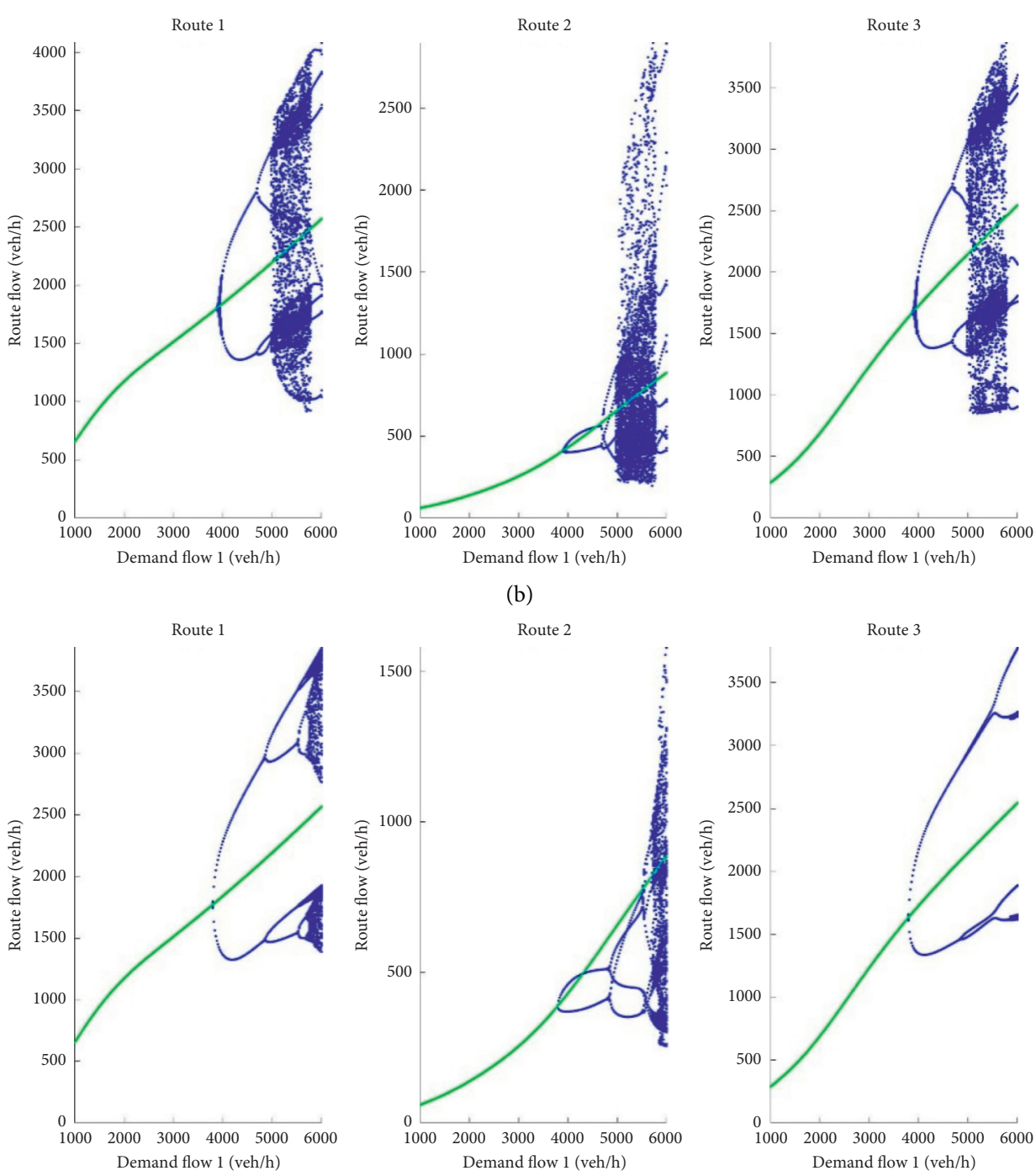

(c)

Figure 2: (a) Bifurcation diagrams of route flows, reference scenario, ES/ES. (b) Bifurcation diagrams of route flows, reference scenario, $\mathrm{MA} / \mathrm{ES} \mu=2$. (c) Bifurcation diagrams of route flows, reference scenario, MA/ES $\mu=3$.

TABLE 4: Bifurcation demand flow, AVs scenarios $\mathrm{A} \theta_{\mathrm{AV}}=7.0$ and $\theta_{\mathrm{AV}}=4.7$

\begin{tabular}{|c|c|c|c|c|c|c|c|c|}
\hline \multirow{2}{*}{$\begin{array}{l}\text { Scenario } \\
\theta_{\mathrm{TV}}=7.0 \\
\theta_{\mathrm{AV}}=4.7\end{array}$} & \multirow{2}{*}{\multicolumn{2}{|c|}{$\mathrm{q}_{m}$}} & \multirow{2}{*}{$\begin{array}{c}\text { DP-ES/ } \\
\text { ES } \\
\text { dBif }\end{array}$} & \multicolumn{5}{|c|}{ DP-MA/ES } \\
\hline & & & & $\begin{array}{c}\mu=2 \\
\mathrm{dBif}\end{array}$ & $\begin{array}{c}\mu=3 \\
\text { dBif }\end{array}$ & $\begin{array}{l}\mu=4 \\
\mathrm{dBif}\end{array}$ & $\begin{array}{l}\mu=5 \\
\text { dBif }\end{array}$ & $\begin{array}{l}\mu=6 \\
\mathrm{dBif}\end{array}$ \\
\hline $\mathrm{A} 1$ & 0.9 & 0.1 & 3958 & 3917 & 3813 & 3958 & 3938 & 3958 \\
\hline $\mathrm{A} 2$ & 0.7 & 0.3 & 4063 & 4000 & 3917 & 4063 & 4021 & 4063 \\
\hline A3 & 0.5 & 0.5 & 4146 & 4104 & 4000 & 4146 & 4125 & 4146 \\
\hline A4 & 0.3 & 0.7 & 4271 & 4229 & 4104 & 4271 & 4229 & 4271 \\
\hline A5 & 0.1 & 0.9 & 4375 & 4333 & 4229 & 4375 & 4354 & 4375 \\
\hline
\end{tabular}

The fixed-point states of both DP model $(11,14)$ and $(13,14)$ are equivalent to the equilibrium states as defined by FP model $(9,10)$. Applying techniques from the theory of discrete-time nonlinear dynamic systems, the above DP models can be used to study the local stability of each fixed-point state, say whether it is an attractor. Moreover, a bifurcation analysis can be carried to single out which attractor is reached by the evolution over time when an input data or a parameter is changed.
The above DP models are a generalization of those already in literature due to the many parameters introduced in the arc flow function to distinguish the vehicle types; see Section 2.4 for further discussion.

2.4. Main Parameters Suitable to Distinguish Vehicle Types. All assignment models discussed above include several parameters useful to distinguish vehicle types; they are 


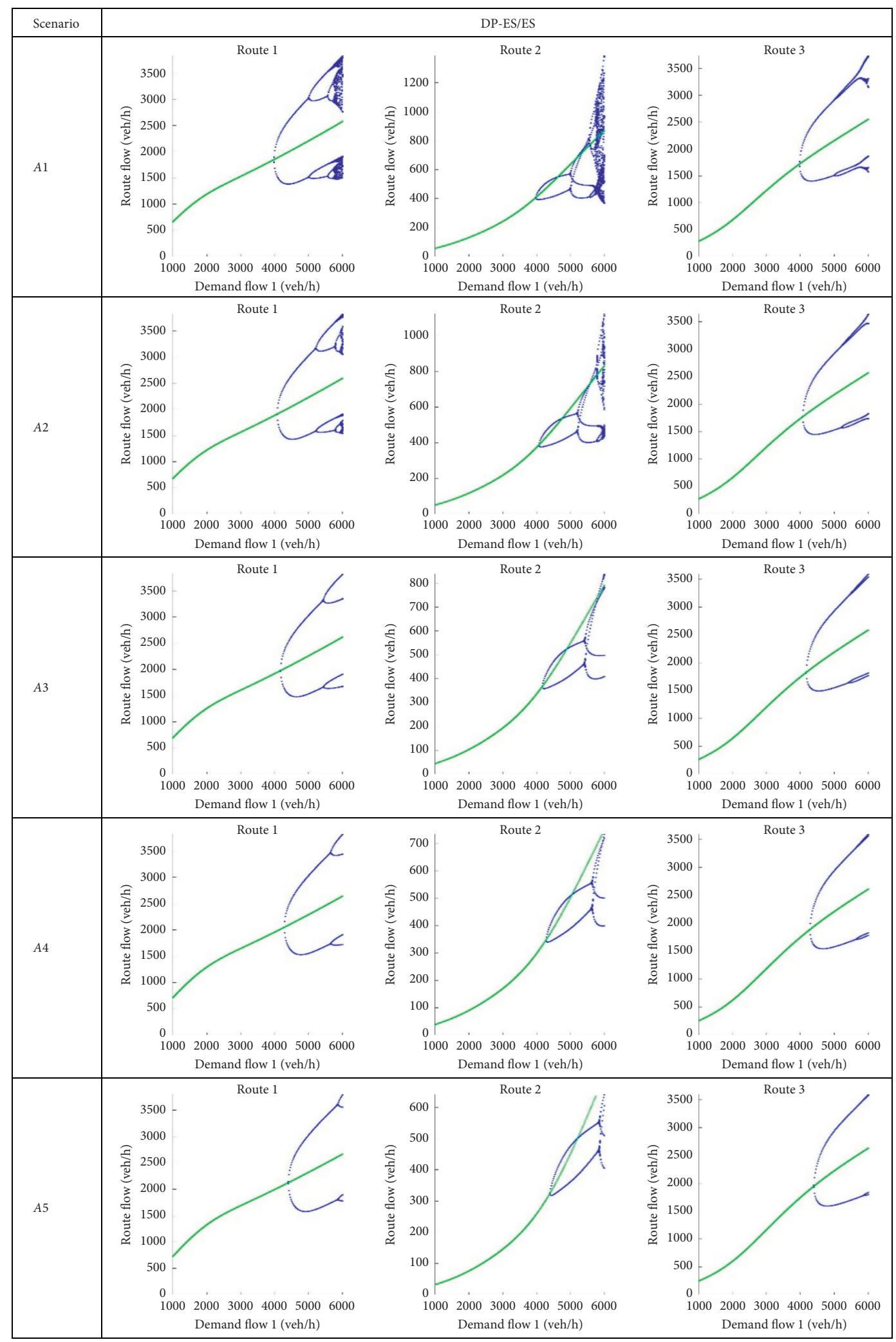

(a)

Figure 3: Continued. 


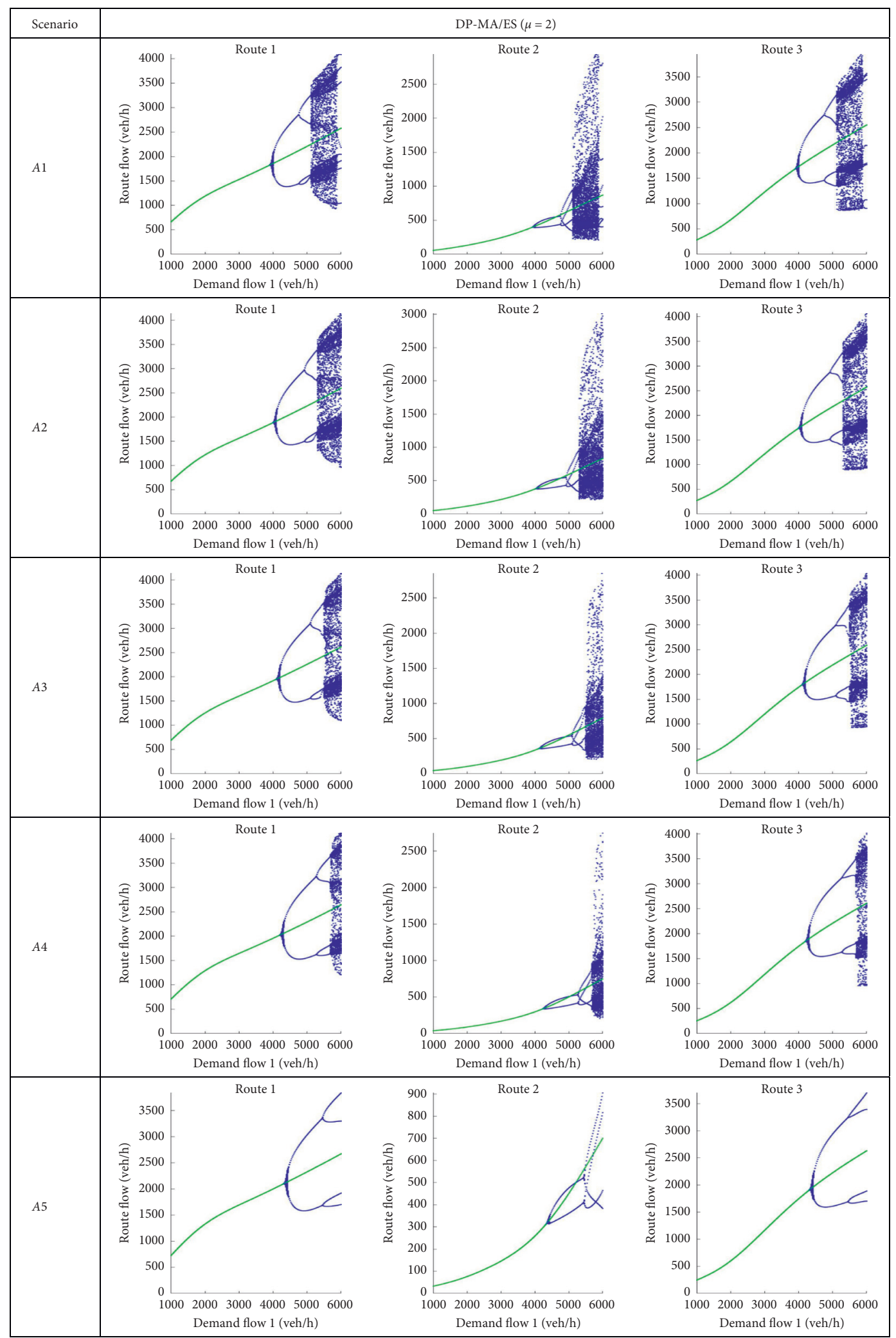

(b)

Figure 3: Continued. 


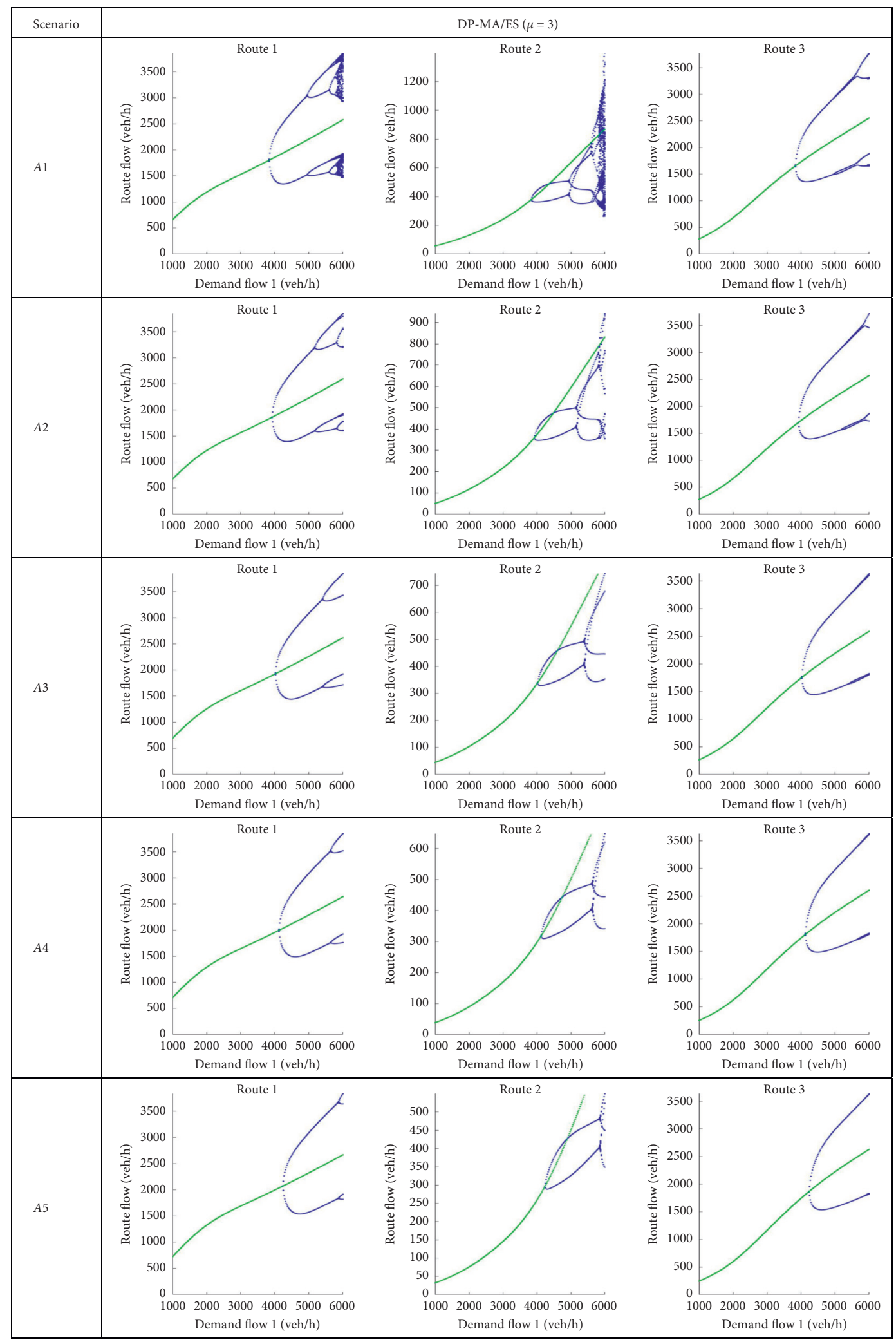

(c)

Figure 3: (a) Bifurcation diagrams of route flows, scenarios A, ES/ES. (b) Bifurcation diagrams of route flows, scenario A, MA/ES $\mu=2$. (c) Bifurcation diagrams of route flows, reference scenario, MA/ES $\mu=3$. 
TABle 5: Bifurcation demand flow, AVs scenarios $\mathrm{B} \theta_{\mathrm{TV}}=7.0$ and $\theta_{\mathrm{AV}}=2.3$

\begin{tabular}{lcccc}
\hline $\begin{array}{l}\text { Scenario } \\
\theta_{\mathrm{TV}}=7.0\end{array}$ & & $\mathrm{q}$ & & DP-ES/ \\
$\theta_{\mathrm{AV}}=2.3$ & TVs & & AVs & ES \\
\hline B1 & 0.9 & & 0.1 & 3833 \\
B2 & 0.7 & 0.3 & 3708 \\
B3 & 0.5 & & 0.5 & 3625 \\
B4 & 0.3 & & 0.7 & 3563 \\
B5 & 0.1 & & 0.9 & 3521 \\
\hline
\end{tabular}

discussed below in detail. As already noted, the reference vehicle type (RVT) has index $m=1$, usually privately owned human-driven fossil fuelled passenger cars.

2.4.1. Supply Parameters. Supply parameters are mainly related to congestion and level-of-service provided. Arc flows as well as arc capacities are measured consistently with arc flows in RVTs per time unit; thus

the flow equivalence coefficient, $\eta_{m}>0$ with $\eta_{1}=1$, is introduced to model different effects of congestion with respect to capacity, for instance, due to average length and/or width, e.g., passenger vs. vans vs. trucks, ...; it is generally assumed that AVs have less effect on congestion than TVs.

It is worth noting that the capacity of $\operatorname{arc} a, \kappa_{a}>0$, say the maximum flow that may traverse $\operatorname{arc} a$, is measured RVTs per time unit; thus its value is independent of the traffic composition, and mostly depends on street features, such as width, slope, etc. In most cost functions, the arc cost actually depends on the ratio between the arc flow and the capacity, $f_{a} / \kappa_{a}$; in this case, the capacity plays the role of arc flow scale factor.

Different types of vehicles may experience different costs on the same arc, thus

the cost equivalence coefficient, $\chi_{m}>0$ with $\chi_{1}=1$, is introduced to model, for example, different speeds, etc. According to the literature, it can be assumed that advanced vehicles may experience smaller generalized costs than TVs, e.g., energy consumption, travel time, and value of time [18-22].

Different types of vehicles may have different arc access allowance, e.g., lanes or areas reserved to e-vehicles, and/or experience different tolls, e.g., congestion tools; thus

the arc specific cost of traversing arc $a$ per vehicle type $m, \mathbf{c}_{\mathrm{Z} m a}$ collected in vector $\mathbf{c}_{\mathrm{Z} m} \geq 0$, is summed to the generic cost affected by congestion, say $\chi_{m} \mathbf{c}_{a}$.

2.4.2. Demand Parameters. Demand parameters are mainly related to user behavior. Users moving with different types of vehicles along the same route may experience different (dis) utility with respect to the same route cost; thus in the utility function

the utility scale parameter, $\psi_{m}>0$ such that the term $\psi_{i}$ $\mathbf{w}_{i}$ is dimensionless, is introduced to model on-board comfort, privacy, etc.; it is generally assumed that AVs experience smaller disutility than TVs since other activities than driving may be carried out when on-board.

Any route choice function, usually but not necessarily derived from Random Utility Theory (RUT), contains some parameters modeling dispersion and other features of the perceived utility distribution that may be different per vehicle type; thus

the route choice function parameter, vector, $\boldsymbol{\theta}_{m}>0$, is introduced; in some models, as the Logit, a scale factor $\theta_{m 1}$ is present, often proportional to the perceived utility dispersion.

As far as calibration is concerned, the utility scale parameter $\theta_{m 1}$ may not be distinguished from the utility parameter $\psi_{m}$; thus their ratio plays the role of a single parameter; on the other hand, the meanings of the two parameters are different and this condition should carefully be taken into account in scenario design.

To convert demand and route flows measured in users per unit of time into arc flows measured in vehicles per unit of time,

the occupancy factor, say the average number of users on-board $\varphi_{m} \geq 1$, is introduced; for example, it is expected that advanced vehicles may allow a higher occupancy factor than other types, for instance, in large-scale ride-sharing with autonomous vehicles.

From the mathematical point of view, each ratio $\left(\eta_{m} / \mu_{m}\right)$ plays the role of a single parameter; on the other hand, the meanings of the two parameters are different and this condition should carefully be taken into account in scenario design.

Vehicle types may also be differentiated with respect to the route choice function $\mathbf{p}_{i m}(\bullet)$.

2.4.3. Dynamic Updating Parameters. Dynamic updating parameters, the choice updating parameter, the cost updating parameter, and the memory depth in MA filters, are related to memory and learning modeling; they might be differentiated per vehicle type. But, in this case the fixed-point stability conditions and bifurcation analysis already in literature (see Cantarella et al. (2019) [14] for details) no longer apply; extension of these results may easily be conceived from the theoretical point of view, but can hardly be derived in practice since involving the closed form solution of high-degree polynomial equations with complex coefficients.

\section{Numerical Examples}

This section discusses the results of the application of the ES/ ES and MA/ES DP models to a toy network to study the effects on fixed-point state stability of introducing AVs.

3.1. Input Data and Assumptions. A toy network with 4 nodes $\mathrm{A}, \mathrm{B}, \mathrm{C}, \mathrm{D}$, and $5 \operatorname{arcs}, 1=(\mathrm{A}, \mathrm{C}), 2=(\mathrm{B}, \mathrm{D}), 3=(\mathrm{B}, \mathrm{C})$, $4=(A, B), 5=(C, D)$, is considered (Figure 1). 


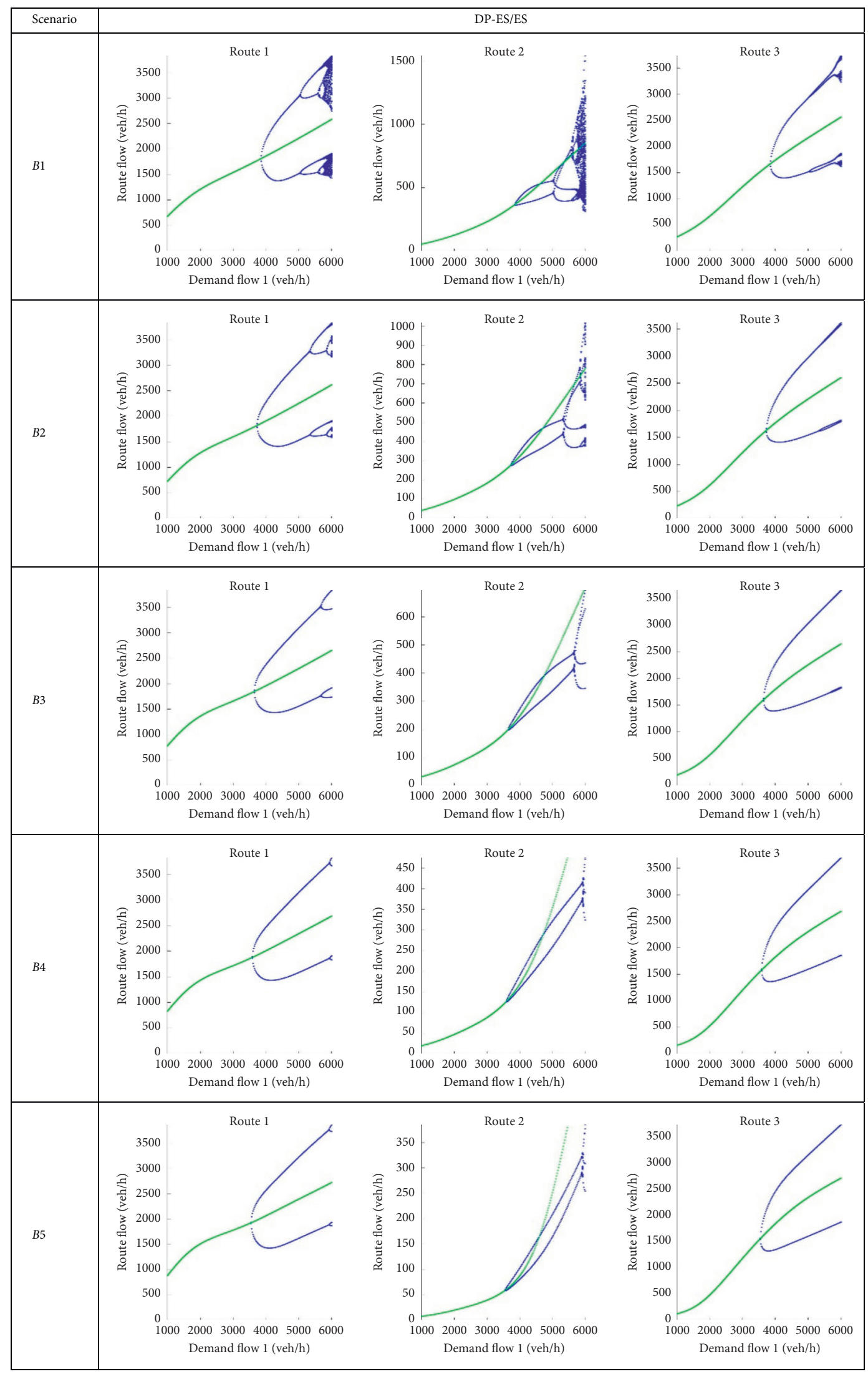

Figure 4: Bifurcation diagrams of route flows, scenarios A, ES/ES with $\theta_{\mathrm{TV}}=7.0$ and $\theta_{\mathrm{AV}}=2.3$. 
TABLE 6: Parameters of vehicle types.

\begin{tabular}{lcc}
\hline Type & $\left(\eta_{m} / \mu_{m}\right)$ & $\chi_{m}$ \\
\hline 1 (TVs) & 1.0 & 1.0 \\
2 (AVs) & 0.7 & 0.8 \\
\hline
\end{tabular}

TABLE 7: Bifurcation demand flow, AVs scenarios C: $\theta_{\mathrm{TV}}=7.0$ and $\theta_{\mathrm{AV}}=4.7 ;\left(\eta_{m} / \mu_{m}\right)=0.7$ and $\chi_{m}=0.8$

\begin{tabular}{lccc}
\hline Scenario & & $\mathrm{q}$ & \\
$\theta_{\mathrm{TV}}=7.0$ & & & DP-ES/ES \\
$\theta_{\mathrm{AV}}=4.7$ & TVs & AVs & \\
$\left(\eta_{m} / \mu_{m}\right)=0.7$ & & & $\mathrm{dBif}$ \\
$\chi_{m}=0.8$ & & 0.1 & 4021 \\
$C 1$ & 0.9 & 0.3 & 4250 \\
$C 2$ & 0.7 & 0.5 & 4500 \\
$C 3$ & 0.5 & 0.7 & 4771 \\
$C 4$ & 0.3 & 0.9 & 5104 \\
$C 5$ & 0.1 & & \\
\hline
\end{tabular}

TABLe 8: Bifurcation demand flow, AVs scenarios $D: \theta_{\mathrm{TV}}=7.0$ and $\theta_{\mathrm{AV}}=2.3 ;\left(\eta_{m} / \mu_{m}\right)=0.7$ and $\chi_{m}=0.8$

\begin{tabular}{lccc}
\hline Scenario & & $\mathrm{q}$ & \\
$\theta_{\mathrm{TV}}=7.0$ & & & DP-ES/ES \\
$\theta_{\mathrm{AV}}=2.3$ & TVs & AVs & \\
$\left(\eta_{m} / \mu_{m}\right)=0.7$ & & & $\mathrm{dBif}$ \\
$\chi_{m}=0.8$ & 0.9 & 0.1 & 3917 \\
$D 1$ & 0.7 & 0.3 & 3938 \\
$D 2$ & 0.5 & 0.5 & 3979 \\
$D 3$ & 0.3 & 0.7 & 4042 \\
$D 4$ & 0.1 & 0.9 & 4125 \\
$D 5$ & & & \\
\hline
\end{tabular}

The pair of nodes $(A, D)$ is the only o-d pair, connected by 3 routes: $1=(A C D), 2=(A B D)$, and $3=(A B C D)$ (paths are numbered after arcs 1,2 , and 3 , respectively)

BPR-like separable arc cost functions are considered (no arc specific cost is considered):

$$
c_{a}=c_{0 \mathrm{a}}\left(1+v_{1}\left(\frac{f_{a}}{\kappa_{a}}\right)^{v_{2}}\right)
$$

where $v_{1}>0$ is the congestion multiplier: how much greater the arc cost is when flow is equal to capacity with respect to zero flow cost; this parameter is to be calibrated against data, in urban applications, 2 is an often used value; $v_{2}>0$ is the congestion exponent: how fast the arc cost increases against flow; this (integer) parameter too is to be calibrated against data; in urban applications, 2 is an often used value; as this value increases, the shape of the function tends to a vertical asymptote; $c_{0 \mathrm{a}}>0$ is the cost when flow is zero.

Costs are given by travel times and measured in minutes; all flows and capacities are measured in vehicles per hour. Values of parameters are given in Table 1.

Given the values of capacities in Table 1, the max flow that can traverse the network from $A$ to $D$ is 6000 . Given the zero flow arc costs, the cost of the shortest path from A to $D$ with is $w_{\mathrm{MINo}}=30$.

User may travel through either of two types of vehicles: TVs (1) and AVs (2). In both cases, the route choice behavior for user travelling is modeled by a Logit choice function:

$$
p_{r}=\frac{\exp \left(v_{r} / \theta_{m}\right)}{\sum_{k \in R i} \exp \left(\mathrm{v}_{k} / \theta_{m}\right)}, \quad \text { for any route } \mathrm{r}
$$

where the dispersion parameter $\theta_{m}>0$ per vehicle type $m$ is proportional to the standard deviation $\sigma$ of the route perceived utility modeled as a random variable, according to the Random Utility Theory. It includes the utility scale parameter $\psi_{m}$.

Vehicle types are also distinguished with respect to ratio $\left(\eta_{m} / \mu_{m}\right)$ and cost equivalent $\chi_{m}$ parameters, with TVs (1) being the RVT, as given in Table 2, where AVs are assumed having a beneficial effect on congestion and being faster.

3.2. Results of a Bifurcation Analysis. To study the effects of introducing of AVs on fixed-point state stability, the results of a bifurcation analysis with respect to the value of demand flow $d$ are presented below varying the vehicle type proportions $q_{m}$ as well as the dispersion parameters $\theta_{m}$.

The evolution over time of the system is described by the DP models in Section 2.3, say ES/ES and MA/ES with $\mu=2,3$, $4,5,6$ to compare the effect of different memory and learning filters. In all scenarios analyzed below, $\alpha=0.5$ and, $\beta=0.6$.

Since the arc cost functions are continuous and monotone increasing and the Logit choice functions are continuous and invariant (dispersion parameter $\theta_{m}$ does not depend on the systematic utility $v_{i m}$ ), there exists exactly one fixed-point state, equivalent to the equilibrium pattern. 


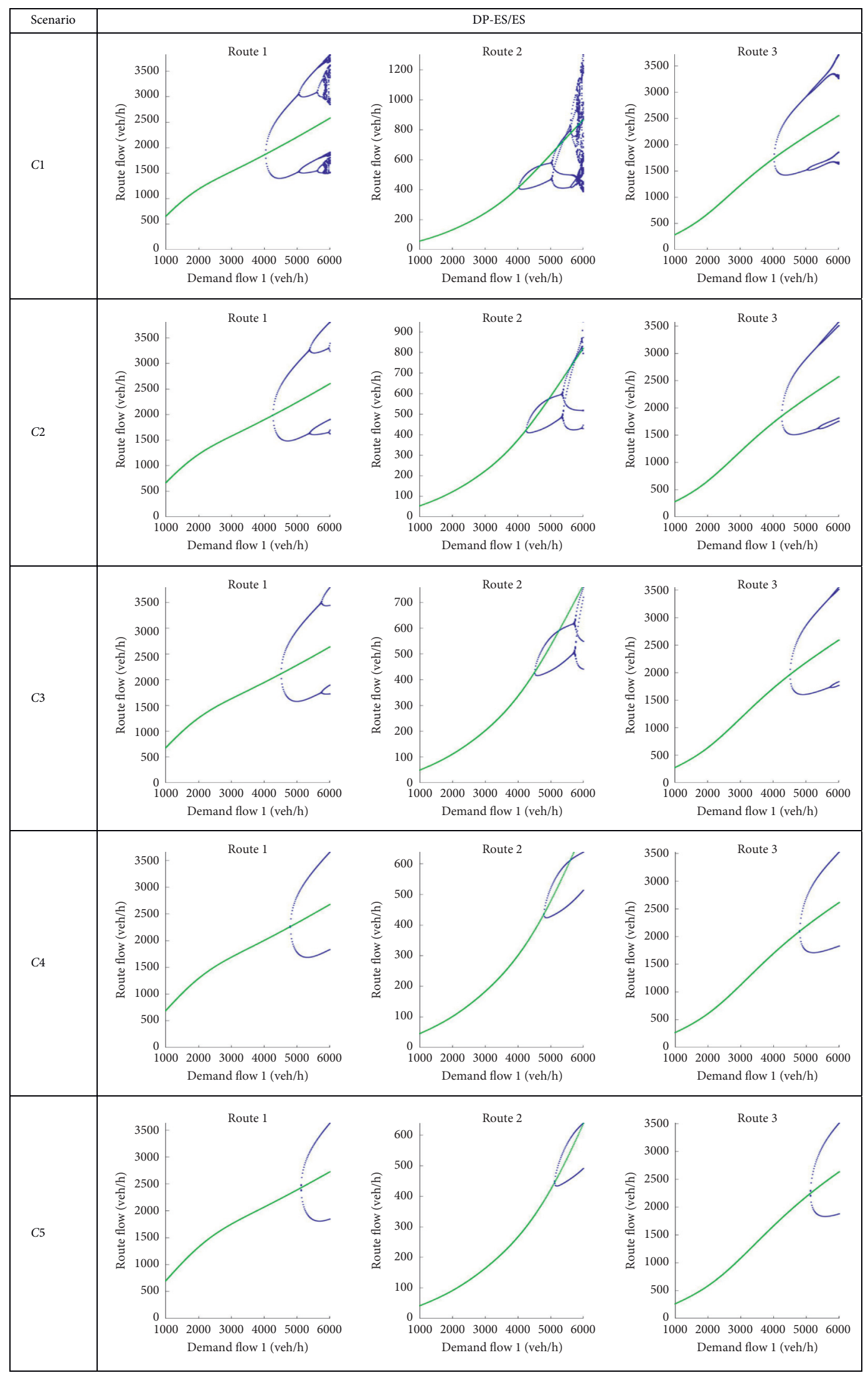

Figure 5: Bifurcation demand flow, AVs scenarios C: $\theta_{\mathrm{TV}}=7.0$ and $\theta_{\mathrm{AV}}=4.7 ;\left(\eta_{m} / \mu_{m}\right)=0.7$ and $\chi \mathrm{m}=0.8$. 


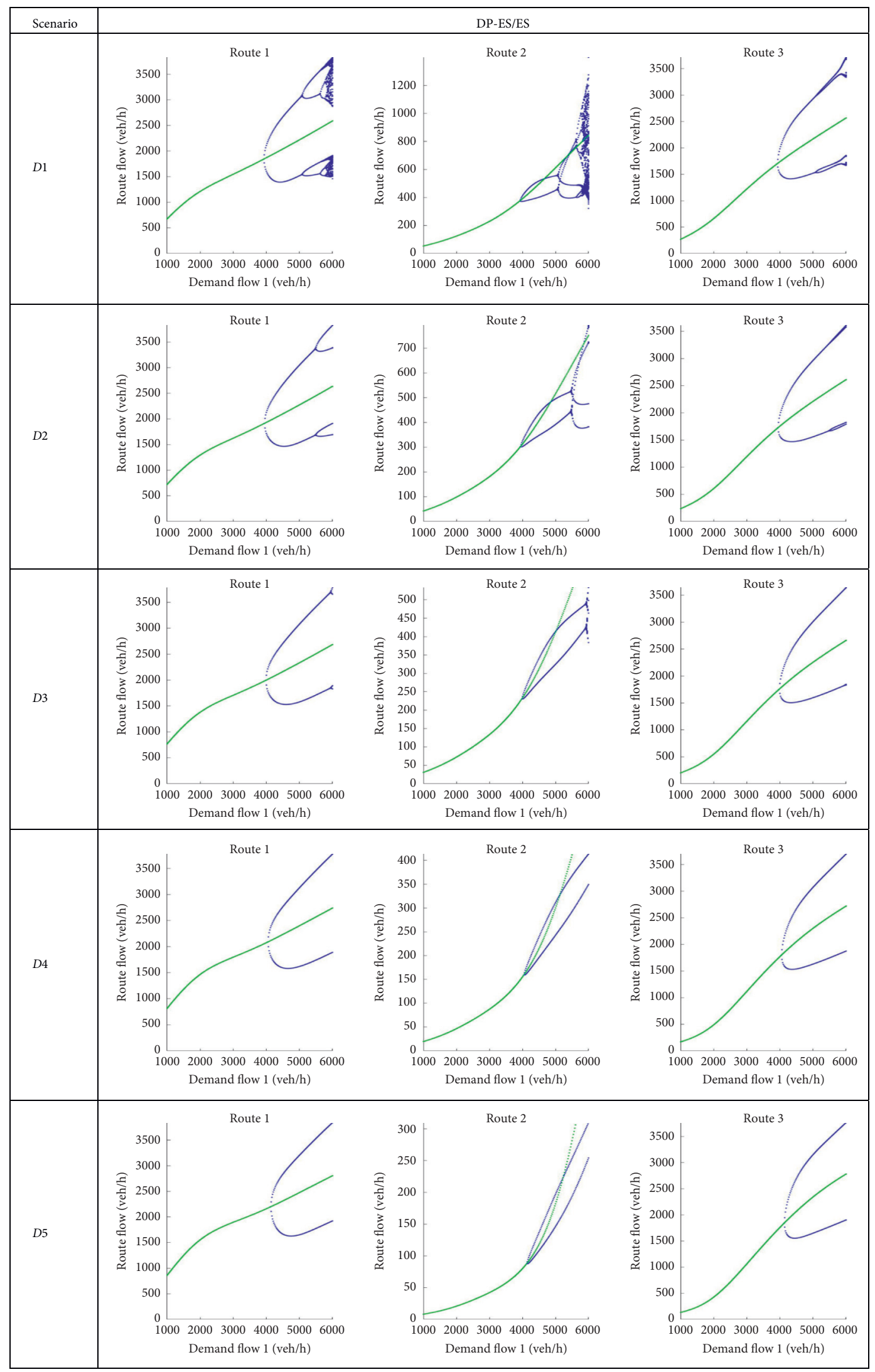

Figure 6: Bifurcation demand flow, AVs scenarios C: $\theta_{\mathrm{TV}}=7.0$ and $\theta_{\mathrm{AV}}=2.3 ;\left(\eta_{m} / \mu_{m}\right)=0.7$ and $\chi \mathrm{m}=0.8$. 
Moreover, it can be proven that flip bifurcations only may occur since the arc cost functions are monotone increasing and separable (see Cantarella et al. 2019 [14]).

3.2.1. Reference Scenario: All TVs. In the reference scenario, only TVs are considered. The dispersion parameter is $\theta_{\mathrm{TV}}=7.0$, corresponding to $30 \%$ variation coefficient with respect to $w_{M I N o}$. Table 3 shows the values of demand flow, $\mathrm{dBif}$, at which the flip bifurcation occurs: the unique fixedpoint stable becomes unstable. The values of $\mathrm{dBif}$ have been found through numerical search.

They have also been verified through the stability conditions based on eigenvalues of the Jacobian of the ES/ES DP model (details in Cantarella et., 2019 [14]). Analogous conditions for the MA/ES DP model are not yet available.

Regarding the memory and learning filters, greater memory depth $\mu$ has a stabilizing effect, as expected, and results for MA tend to ES as the $\mu$ increases. Rather surprisingly, the convergence of the results from the MA filter to those from the ES filter as the memory depth $\mu$ increases is not smooth but dBif oscillates between odd and even values, $\mathrm{dBif}$ for an odd value of $\mu$ being smaller than the $\mathrm{dBif}$ for the two near even values. This condition is worth further research work.

For values of demand flow greater than dBif, the system evolves towards a 2-periodic attractor as shown by bifurcation diagrams (Figure 2) with respect to values of demand flow up to 6000 for ES and MA with $\mu=2$ or 3 only, the green line showing the equilibrium route flow.

3.2.2. Scenarios $A$ and $B$ : Increasing Proportion of $A V s$. In scenarios $A$, the effect of increasing the AVs proportion is analyzed. The dispersion parameter of TVs is still $\theta_{\mathrm{TV}}=7.0$, but the dispersion parameter of AVs is $\theta_{\mathrm{AV}}=4.7$, corresponding to $20 \%$ variation coefficient with respect to $w_{M I N o}$, since AVs may have access to information reducing uncertainty both of users and modelers. Table 4 shows the values of demand flow, dBif, at which the flip bifurcation occurs. Bifurcation diagrams are reported in Figure 3; as expected, flip bifurcations occur towards periodic and aperiodic attractors.

Increasing the AVs proportion has a positive effect on fixed-point stability, since the bifurcation demand flow increases reducing the set of values of demand flow for which the fixed point is not stable. Indeed, AVs have less effect on congestion as modeled by the flow equivalent flow coefficient (including the occupancy factor) and smaller costs as modeled by the cost equivalent parameter. In all scenarios, great memory depth $\mu$ has a stabilizing effect.

The beneficial effect of AVs may vanish if the dispersion among users is reduced to $\theta_{\mathrm{AV}}=2.3$, corresponding to $10 \%$ variation coefficient with respect to $w_{M I N o}$. Table 5 shows, for ES/ES DP only, that the values of demand flow dBif at which the flip bifurcation occurs reduce as the AVs proportion increases, increasing the values of demand flow for which the fixed point is not stable. Indeed, reducing dispersion may lead fixed-point states towards instability. Bifurcation diagrams are reported in Figure 4.
3.2.3. Scenarios $C$ and D: Decreasing AVs Vehicle Type Parameters. In these scenarios, the vehicle types parameters for AVs have been reduced. In Table 6, the values of the AVs parameters adopted have been reported.

The DP-ES-ES cases have been analyzed with these new parameters both for $\theta \mathrm{AV}=4.7$ (Scenario C) that for $\theta \mathrm{AV}=2.3$ (Scenario D). Results are shown in Tables 7 and 8 for Scenario C and Scenario D, respectively.

In Scenario $C$, the same general trend observed in Scenario A (where the same $\theta_{\mathrm{AV}}=4.7$ is used) can be observed (i.e., increasing the AVs proportion has a positive effect on fixed-point stability, since the bifurcation demand flow increases reducing the set of values of demand flow for which the fixed point is not stable). In comparison with Scenario A, higher values of $\mathrm{dBif}$ can be observed in this Scenario C; thus, reducing the AVs parameters of vehicle types, a positive effect can be observed on the system. Similar results are highlighted comparing Scenario D (see Table 8) with Scenario $\mathrm{B}$, where the same $\theta_{\mathrm{AV}}=2.3$ is adopted.

Bifurcation diagrams, for Scenarios C and D, are reported in Figures 5 and 6.

\section{Conclusions}

In this paper, deterministic process models for day-to-day dynamic multivehicle assignment, including fixed-point models for equilibrium assignment as a special case, have been analyzed.

Furthermore, results of an application to a toy network are discussed and a comparison has also been carried out between moving average and exponential smoothing filters for modeling user memory and learning. Similar results may be obtained through bifurcation analysis with respect to other parameters, such the occupancy factor or the updating parameters $\alpha$ and $\beta$.

Generally, AVs have less impact on congestion than TVs do, but, even on a very simple network application resulted in that AVs may not have a positive effect of equilibrium stability since negative effect of reducing dispersion may be greater than positive effects on congestion.

To apply the proposed methodological framework to a real case-study (network and vehicle characteristics), a parameter calibration against real data is required. However, as of today, proper data are not available in the literature.

Worthy of further research efforts is the application of the proposed framework to real-world cases that will become increasingly important in the near future to evaluate the impact of new vehicle types, and stability conditions for MA/ ES DP with $\mu=2$. Moreover, a detailed and effective modeling of the effects of shared vehicles with public access, socalled robotaxi, is still an open issue.

\section{Data Availability}

No real data have been used in the paper. Theoretical findings are grounded on simulation experiments in a laboratory network. 


\section{Conflicts of Interest}

The authors declare that there are no conflicts of interest regarding the publication of this paper.

\section{Acknowledgments}

The authors thank M. Di Gangi and O. Giannattasio for several stimulating conversations about the topics of this paper. The authors also thank University of Salerno for financial support under local grants ORSA192198 and ORSA208090 and under the Italian Program PON AIMAttraction and International Mobility, Linea 1 (AIM1877579-3-CUP-D44I18000220006).

\section{References}

[1] J. G. Wardrop, "Road paper. Some theoretical aspects OF road traffic research," Proceedings of the Institution of Civil Engineers, vol. 1, no. 3, pp. 325-362, 1952.

[2] C. F. Daganzo and Y. Sheffi, "On stochastic models of traffic assignment," Transportation Science, vol. 11, no. 3, pp. 253-274, 1977.

[3] C. F. Daganzo, "Stochastic network equilibrium with multiple vehicle types and asymmetric, indefinite link cost jacobians," Transportation Science, vol. 17, no. 3, pp. 282-300, 1983.

[4] G. E. Cantarella, "A general fixed-point Approach to multimode multi-user equilibrium assignment with elastic demand," Transportation Science, vol. 31, no. 2, pp. 107-128, 1997.

[5] J. L. Horowitz, "The stability of stochastic equilibrium in a two-link transportation network," Transportation Research Part B Methodology, vol. 18, no. 1, pp. 13-28, 1984.

[6] G. E. Cantarella, "Day-to-day dynamic models for intelligent transportation systems design and appraisal," Transportation Research Part C: Emerging Technologies, vol. 29, pp. 117-130, 2013.

[7] G. E. Cantarella and D. P. Watling, "Modelling road traffic assignment as a day-to-day dynamic, deterministic process: a unified approach to discrete- and continuous-time models," EURO Journal on Transportation and Logistics, vol. 5, no. 1, pp. 69-98, 2016.

[8] E. Cascetta, Static and Dynamic Models of Stochastic Assignment to Transportation Networks, Flow Control Of Congested Networks, Berlin, Germany, pp. 91-111, 1987.

[9] E. Cascetta, "A stochastic process approach to the analysis of temporal dynamics in transportation networks," Transportation Research Part B Methodology, vol. 23, no. 1, pp. 1-17, 1989.

[10] G. E. Cantarella and E. Cascetta, "Dynamic processes and equilibrium in transportation networks: towards a unifying theory," Transportation Science, vol. 29, no. 4, pp. 305-329, 1995.

[11] G. E. Cantarella and D. P. Watling, "A general stochastic process for day-to-day dynamic traffic assignment: formulation, asymptotic behaviour, and stability analysis," Transportation Research Part B: Methodological, vol. 92, pp. 3-21, 2016.

[12] D. P. Watling and G. E. Cantarella, "Model representation \& decision-making in an ever-changing world: the role of stochastic process models of transportation systems," Networks and Spatial Economics, vol. 15, no. 3, pp. 843-882, 2015.
[13] D. P. Watling and M. L. Hazelton, "Asymptotic approximations of transient behaviour for day-to-day traffic models," Transportation Research Part B: Methodological, vol. 118, pp. 90-105, 2018.

[14] G. E. Cantarella, D. P. Watling, S. de Luca, and R. Di Pace, Dynamics and Stochasticity in Transportation Systems: Tools for Transportation Network Modelling, Elsevier, New York, NY, USA, 2019.

[15] G. E. Cantarella and A. D. Febbraro, "Transportation Systems with Autonomous Vehicles: models and algorithms for equilibrium assignment," Transportation Research Procedia, vol. 27, pp. 349-356, 2017.

[16] G. E. Cantarella, A. D. Febbraro, M. D. Gangi, and O. Giannattasio, "Solving stochastic assignment to transportation networks with TVs and AVs," Transportation Research Procedia, vol. 42, pp. 7-18, 2019.

[17] G. E. Cantarella, A. D. Febbraro, M. D. Gangi, and O. Giannattasio, "Stochastic multi-vehicle assignment to urban transportation networks," in Proceedings of the 2019 6th International Conference On Models And Technologies For Intelligent Transportation Systems (MT-ITS), pp. 1-8, Kraków, Poland, 2019.

[18] L. Kröger, T. Kuhnimhof, and S. Trommer, "Does context matter? A comparative study modelling autonomous vehicle impact on travel behaviour for Germany and the USA," Transportation Research Part A: Policy and Practice, vol. 122, pp. 146-161, 2019.

[19] M. W. Levin and S. D. Boyles, "Effects of autonomous vehicle ownership on trip, mode, and route choice," Transportation Research Record: Journal of the Transportation Research Board, vol. 2493, no. 1, pp. 29-38, 2015.

[20] S. Esmaeilzadeh Seilabi, M. Tarighat Tabesh, A. Davatgari, M. Miralinaghi, and S. Labi, "Promoting autonomous vehicles using travel demand and lane management strategies," Frontiers in Built Environment, vol. 6, p. 156, 2020.

[21] Z. Chen, F. He, Y. Yin, and Y. Du, "Optimal design of autonomous vehicle zones in transportation networks," Transportation Research Part B: Methodological, vol. 99, pp. 44-61, 2017.

[22] Z. Chen, F. He, L. Zhang, and Y. Yin, "Optimal deployment of autonomous vehicle lanes with endogenous market penetration," Transportation Research Part C: Emerging Technologies, vol. 72, pp. 143-156, 2016. 